\title{
NON-COHERENT MASSIVE MIMO-OFDM FOR COMMUNICATIONS IN HIGH MOBILITY SCENARIOS
}

\author{
Kun Chen- $\mathrm{Hu}^{1}$, Yong Liu ${ }^{2}$, Ana Garcia Armada ${ }^{1}$ \\ ${ }^{1}$ Deparment of Signal Theory and Communications of Universidad Carlos III of Madrid (Spain). E-mails: \{kchen, \\ agarcia\}@tsc.uc3m.es, ${ }^{2}$ Shanghai Research Center, Huawei Technologies (China). E-mail: liu.liuyong@huawei.com.
}

\begin{abstract}
Under scenarios of high mobility, the traditional coherent demodulation schemes (CDS) have a limited performance, due to the fact that reference signals cannot effectively track the variations of the channel with an affordable overhead. As an alternative solution, non-coherent demodulation schemes (NCDS) based on differential modulation have been proposed. Even in the absence of reference signals, they are capable of outperforming the CDS with a reduced complexity. The literature on NCDS laid the theoretical foundations for simplified channel and signal models, often single-carrier and spatially uncorrelated flat-fading channels. In this work, the most recent results assuming orthogonal frequency division multiplexing (OFDM) signaling and realistic channel models are explained, and the impact of some hardware impairments such as the phase noise (PN) and the non-linear high power amplifier (HPA) are also considered. Moreover, new potential research lines are also highlighted.
\end{abstract}

Keywords - 5G, channel estimation, MIMO, non-coherent, OFDM.

\section{INTRODUCTION}

The Fifth Generation of mobile communications (5G) [1] is the global standard for a unified wireless air interface, which is capable of providing a great flexibility for a multitude of use cases. The three main requirements of those services are enhanced mobile broadband (eMBB), massive machine type communications (mMTC) and ultra reliable low-latency communications (URLLC). Therefore, the peak data-rate is not the only feature to be improved, but also an enormous number of connected devices and the latency-sensitive services are taken into account. Also there is an increasing interest in providing an adequate service in high mobility scenarios [2] - [4].

Orthogonal frequency division multiplexing (OFDM) with multiple-input multiple-output (MIMO) [5] have been recently set as the radio techniques for the physical layer in 5G [1]. New frequency bands are proposed to be exploited to obtain more available bandwidth, such as $3.5 \mathrm{GHz}$ and millimeter-waves (mm-Wave) [6], and thus, the existing services can be improved and new ones can be implemented. The integration of massive MIMO is a must, not only to improve the average capacity of the link, but also for the implementation of beam-steering and beamforming to mitigate propagation losses in these new higher bands. Furthermore, the complexity of the signal processing techniques need to be bounded to reduce the cost of the devices and the delay of the required operations. As an alternative to classical coherent demodulation schemes (CDS), non-coherent demodulation schemes (NCDS) [7-9] have been proposed recently to be combined with massive MIMO systems [10] - [19]. They are capable of avoiding the overhead produced by the reference signals due to the fact that the transmitted symbols can be recovered without the knowledge of channel state information (CSI). This overhead can be excessively high for very fast time-varying channels. In such cases, a significant number of reference signals is required for the continuous tracking of the channel estimation [20].

The works in the literature have provided the theoretical foundations to understand NCDS and point to some cases when they can outperform the traditional CDS, in particular in scenarios with high mobility [20]. Also, recent works show some combinations of NCDS with MIMOOFDM for the uplink (UL) and downlink (DL). In the present work, convinced that NCDS is an idea whose time has come, we discuss the implementation of the NCDS in practical MIMO-OFDM communication systems, assuming some realistic channels characterized by high mobility. We provide the details of how to integrate the differential modulation [21] in the two-dimensional resource grid (time and frequency) provided by the OFDM. Additionally, we also show the performance of this combination under the effects of the phase noise (PN) [22], [23] or high power amplifier (HPA) [24], for both UL and DL, and its benefits as compared to the traditional CDS. Finally, a discussion related to challenges and opportunities is provided together with some concluding remarks, in order to stimulate the research on this promising topic.

The remainder of the paper is organized as follows. Section 2 introduces the main differences between CDS and NCDS, especially for high mobility scenarios. Section 3 and Section 4 provide the details of how to integrate the NCDS with MIMO-OFM for the UL and DL, respectively. Section 5 presents several numerical results to evaluate the proposed scheme under some realistic channel models, providing an assessment of the achieved system performance. Finally, in Section 6, the conclusions follow. 


\section{BENEFITS AND WEAKNESSES OF THE CO- HERENT AND NON-COHERENT DEMOD- ULATION SCHEMES}

\subsection{Coherent demodulation schemes (CDS)}

Well-known coherent detection requires a replica of the carrier at the receiver, with frequency and phase synchronized, with the transmitted one, and an estimation of the channel attenuation and phase. Then, the received signal and a replica of the received version of all possible transmitted signals can be cross-correlated to make a decision. CDS are widely used by many communication systems. In particular, they are used in 5G [1], where the advantages of MIMO-OFDM are exploited, providing a high throughput through the use of the well-known $M$-ary quadrature amplitude modulation (QAM). With this modulation format, the information is transported in both the amplitude and phase of the carrier, making an efficient use of the transmission channel. However, these benefits come at the expense of transmitting some reference signals in order to obtain accurate enough CSI, so that the effects produced by the propagation channel to the received symbols can be equalized before a decision. When the channel is frequency-selective, OFDM facilitates the implementation of CDS due to the fact that each subcarrier can be considered as having an independent flat-fading channel, reducing the complexity of the equalization.

The need to obtain accurate enough CSI is accepted in most communication systems, in particular when the channel impulse response remains quasi-static for a certain period of time and the number of antennas is not very large. Under these conditions, a reduced amount of reference signals are used in order to track the channel variations in time, frequency and space dimensions. On the other hand, if we would like to provide communications in new challenging environments, such as high speed trains, autonomous vehicles, etc. these are mainly characterized by a significant Doppler spread due to the high mobility. In these situations, the traditional CDS requires an enormous amount of reference signals in order to continuously and accurately track the variations of the channel, reducing considerably the overall efficiency of the system, as pointed out in [10], [11], [20]. Otherwise, if the CSI is not properly estimated, the performance of the CDS is also seriously compromised. Moreover, when massive MIMO is considered, the procedures of channel estimation and the computation of the pre/post-coding matrices may increase the complexity of the system. For example, the channel inversion of large dimension matrices for each subcarrier may be prohibitive for some real-time applications when a zero-forcing (ZF) criterion is chosen.

\subsection{Non-Coherent demodulation schemes (NCDS)}

Looking back in history, NCDS are older than CDS. In [7], a comparison is made of the output spectra comprising signal and low-frequency noise when a sinusoidal signal plus noise is applied to several types of detector. It is shown that a considerable gain may be obtained by using the (new at that time) coherent detector as compared to the non-coherent square-law detector when the input signal to noise ratio (SNR) is low. In [8], a complete theory of detection is presented for threshold reception, which requires either a suitably weighted cross-correlation of the received data with the a priori known signal (CDS), or a suitably weighted autocorrelation of the received data with itself (NCDS). The Kineplex system developed by Collins Radio Company introduced the technique of differential phase shift keying (DPSK), as described in [9]. Today, NCDS have been re-proposed as an alternative to the traditional CDS due to the fact that they are able to recover the transmitted symbols without any CSI, that is, knowledge of the amplitude and phase of the carrier is not required. Hence, reference signals are no longer needed, reducing the undesirable signalling overhead. This effect is more relevant for high mobility and/or very frequencyselective scenarios. Additionally, the complexity of the transceivers is significantly reduced. Typical approaches involve the detection of the signal energy of phase differences. Despite its simplicity, non-coherent detection usually implies a $3 \mathrm{~dB}$ loss in SNR as compared to CDS. For this reason, it has just been used in a few communication systems where low complexity was a primary requirement. Examples of application are Bluetooth [25], with a non-coherent frequency shift keying (FSK) receiver or Zigbee [26], using DPSK.

Differential modulation is one of the most frequently used techniques for NCDS [12] - [19]. In this case the information is encoded by computing the phase difference between the current complex data symbol and the previously transmitted symbol. At the receiver, a simple differential decoder is required, detecting the phase difference between two contiguous symbols. To apply this technique, the constellation is constrained to have a constant modulus, such as DPSK, and a single reference symbol is needed at the beginning of each stream to have an initial phase reference. This means a negligible overhead to the system. It is also required that the channel response of every two contiguous differential symbols should be very similar, otherwise the differential decoder is not able to successfully recover the transmitted information data. This condition is usually met, even in fast varying channels.

Recently, the combination of NCDS with massive MIMO has been proposed in order to improve its performance leveraging the high number of antennas. In the context of UL, [10], [11] showed that asymptotically NCDS can achieve the same performance as CDS. Nevertheless, the proposed technique that illustrated this idea required a very large number of antennas to get an acceptable performance. Then, [12] - [16] proposed the use of DPSK together with an averaging process performed at the base station (BS) over the spatial domain after non-coherent demodulation, in order to mitigate the effects of the chan- 
nel and noise. Moreover, they proposed the idea of multiplexing the data of each user equipment (UE) in the constellation domain based on a joint-symbol, which is a superposition of the symbols sent by several UEs. In the case of DL, the combination of NCDS with MIMO has been until now based on block codes [27] - [31]. However, their application requires that the channel response remains quasi-static during the transmission of a block code, and they also need a high SNR in order to provide an acceptable performance. Moreover, they have the problem that they are not scalable and when the number of antennas at the BS is very large, the design of these block codes becomes unaffordable. Typically, only two and four transmit antennas are taken into account [27] - [31]. More recently, the combination of beamforming and NCDS has been proposed in order to exploit the high number of antennas through compensating the path loss and enhancing the quality of the link, and spatially multiplexing the different UEs [18], [19]. In these cases a certain channel knowledge is needed to point the beam towards the UE through the beam-management procedure, and the signal is processed non-coherently in each beam afterwards. Even though the overhead is not completely eliminated, the savings are considerable.

\section{NCDS WITH MASSIVE MIMO FOR THE UP- LINK}

We describe in this section how to integrate the NCDS based on [12] - [17] in a realistic communication system for the particular scenario of UL. We consider one BS equipped with $V$ antennas, which is simultaneously serving $U$ UEs. These UEs are constrained to have a reduced number of antennas, typically single-antenna devices. Let us assume that the $U$ UEs are simultaneously transmitting $N$ OFDM symbols. The OFDM signal has $K$ subcarriers, and the length of the cyclic prefix (CP) is long enough to absorb the effects of the multi-path channel. At the receiver side, after removing the $\mathrm{CP}$ and performing a fastFourier transform (FFT) to each block at each antenna of the BS, we can process each subcarrier as one of a set of $K$ independent subchannels.

\subsection{Integration of differential encoding in OFDM for high mobility scenarios}

Similar to CDS, the NCDS can be also implemented in an OFDM system [17], suitable for dealing with a doublydispersive channel. The stream of differential symbols produced by the differential encoding can be mapped in the two-dimensional resource grid provided by the OFDM (time and frequency). According to [17], the way this mapping is performed will significantly impact on the overall system, especially for high mobility scenarios.

Typically, NCDS based on differential modulation is performed using the time domain scheme. This scheme is represented in Fig. 1, where the red arrows indicate the direction in which differential modulation and demodula-

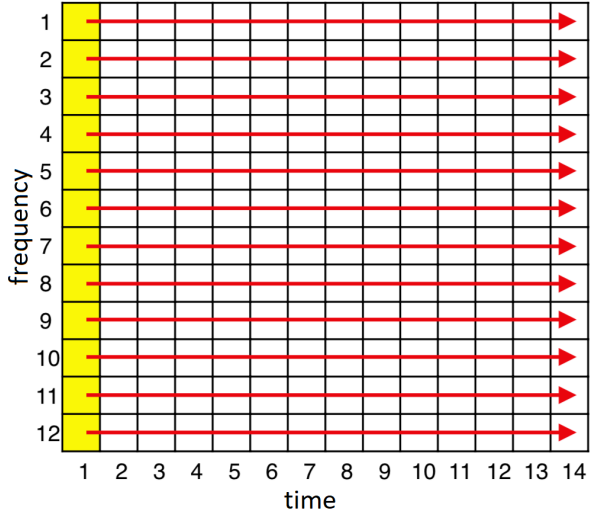

Fig. 1 - Time domain scheme in the OFDM resource grid when $K=12$ and $N=14$. The yellow box represents a reference symbol required by the differential modulation.

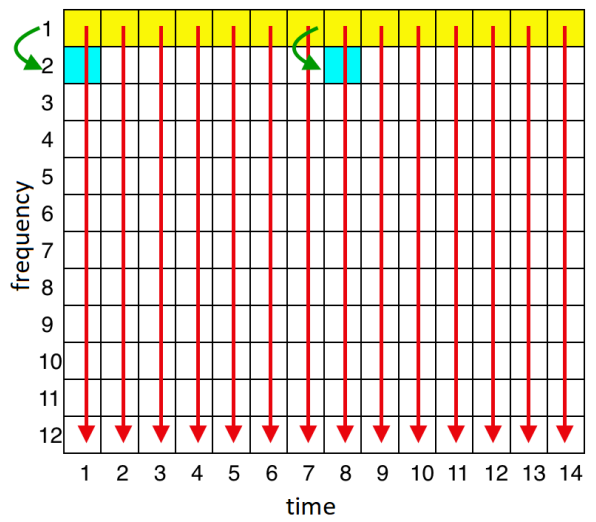

Fig. 2 - Frequency domain scheme in the OFDM resource grid when $K=12, N=14$ and $\mathcal{J}_{N}=\{1,8\}$. The yellow and blue boxes denote the reference symbols required by the differential modulation and phase difference estimation, respectively.

tion is performed, in this case between resources that belong to the same frequency and contiguous symbols in the time domain. The differential encoding can be described as

$$
\begin{gathered}
x_{k, n}^{u}=\left\{\begin{array}{cl}
r_{k, n}^{u}, & n=1 \\
x_{k, n-1}^{u} s_{k, n-1}^{u}, & 2 \leq n \leq N
\end{array},\right. \\
1 \leq u \leq U, \quad 1 \leq k \leq K,
\end{gathered}
$$

where $r_{k, 1}^{u}$ is the reference symbol transmitted at the $k$ th subcarrier of the first OFDM symbol by the $u$-th UE, $s_{k, n}^{u}$ and $x_{k, n}^{u}$ are the complex data and differential symbol, respectively, transmitted at the $k$-th subcarrier and $n$-th OFDM symbol by the $u$-th UE. The data symbol $s_{k, n}^{u}$ belongs to a PSK constellation due to the fact that the differential encoding can only transmit information in the phase component. However, this time-domain implementation has the drawback of an increased latency and memory consumption, since this mapping scheme requires waiting for the reception of two complete OFDM symbols in order to obtain $s_{k, n}^{u}$, due to the fact that it performs a differential decoding of two contiguous symbols in the time domain. Also, it cannot be exploited when the Doppler shift is very high, since any two consecutive OFDM symbols will not face a similar channel response. 
Alternatively, the OFDM frame enables exploiting the frequency dimension, and hence, the differential modulation technique can be also implemented using the frequency domain scheme (see Fig. 2). According to [17], the differential symbols are mapped into contiguous frequency resources of the same OFDM symbol as

$$
\begin{gathered}
x_{k, n}^{u}=\left\{\begin{array}{cl}
r_{k, n}^{u}, & k=1, \\
x_{k-1, n}^{u} p_{k, n}^{u}, & k=2, \quad n \in \mathcal{J}_{N}, \\
x_{k-1, n}^{u} s_{k-1, n}^{u}, & \text { otherwise }
\end{array}\right. \\
1 \leq u \leq U, \quad 1 \leq n \leq N,
\end{gathered}
$$

where $r_{1, n}^{u}$ and $p_{2, n}^{u}$ are two reference symbols for different purposes, and the set $\mathcal{J}_{N}$ contains the indexes that correspond to those OFDM symbols which carry $p_{2, n}^{u}$. The first kind of reference symbol is required for the differential demodulation as explained before. The second one is required for the estimation of the phase difference between two subcarriers, consequence of the frequencydomain mapping; see [17] for more details. We can see that this scheme has a reduced latency and is robust against high Doppler shifts. Furthermore, it is reasonable to assume that the channel responses of any two contiguous subcarriers are similar due to the fact that the number of subcarriers is always designed to be much larger than the number of taps of the channel. However, these benefits come at the expense of an additional phase estimation and compensation procedure. This additional phase component is very small and consequently can be neglected for channels that are not very frequency-selective. On the other hand, this phase must be compensated for strong frequency-selective channels. However, when diversity is exploited, only an additional reference pilot is required for all OFDM symbols within the coherence time $\left(p_{2, n}^{u}\right)$, which produces a negligible impact on overhead.

Both time and frequency domain schemes, presented in [17], may introduce a significant overhead, if the number of allocated resources is reduced $(K \downarrow$ and/or $N \downarrow)$. For example, in scenarios of mMTC, the machine devices are designed to send short packets of just a few bytes. The adoption of any of the two presented schemes implies to send a significant amount of reference symbols. Hence, we propose a new mapping scheme named as mixed domain scheme (see Fig. 3). Firstly, we differentially encode the data symbols as

$$
x_{j}^{u}=\left\{\begin{array}{cl}
r_{j}^{u}, & j=1 \\
x_{j-1}^{u} p_{j}^{u}, & j=2 \\
x_{j-1}^{u} s_{j-1}^{u}, & 3 \leq j \leq K N
\end{array} \quad, \quad 1 \leq u \leq U\right.
$$

where the $j$ denotes the resource index. Then, the differential symbols $x_{j}^{u}$ are allocated to the two-dimensional resource grid as

$$
x_{k, n}^{u}=x_{j}^{u} \mid(k, n)=f(j), \quad 1 \leq j \leq K N,
$$

where $f(\bullet)$ is the resource mapping policy function. Fig. 3 shows a recommended example of a mapping policy

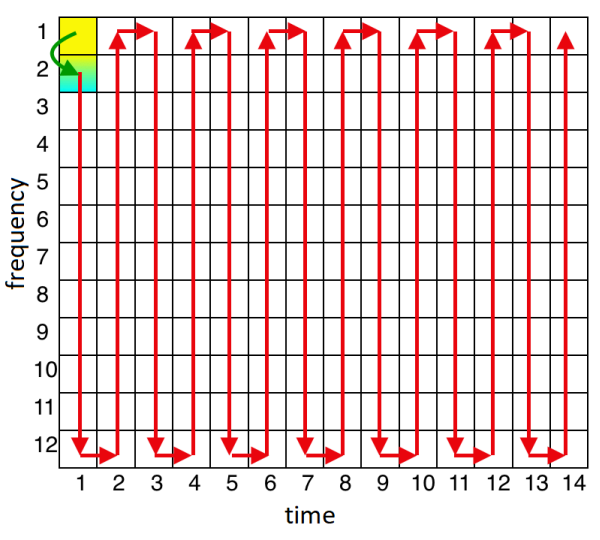

Fig. 3 - Mixed domain scheme in the OFDM resource grid when $K=12$ and $N=14$. The yellow and blue boxes denote the reference symbols required by the differential modulation and phase difference estimation, respectively.

function, where the dramatic reduction of reference signals can be observed. This policy mainly follows the frequency domain scheme, except for the edge subcarriers of the block, that follow a time domain scheme. This proposal cannot only significantly reduce the number of reference symbols, but it is also capable of taking all advantages of a frequency domain scheme. Moreover, in the case of time-varying channels, only those complex symbols placed at both edge subcarriers may suffer from an additional degradation, that can be easily mitigated by using some channel coding [16], [32] or spreading [33] techniques.

For the sake of conciseness and to ease the notation, the frequency domain scheme is the chosen one for the rest of the paper. Note that any of the presented techniques in the following sections can be straightforwardly adopted for both time and mixed domain schemes.

\subsection{Multi-user multiplexing in the constella- tion domain}

For a single-user case, the use of a constant modulus constellation, such as DPSK [12] - [15], is the only requirement for the non-coherent demodulation based on differential detection. However, when a multi-user scenario is considered, if we would like that all independent transmit sources are transmitting in the same time-frequency resource (to increase the spectral efficiency), the received signals from these independent sources are summed up and need to be conveniently separated [12]. Then, the choice of the constellation for each individual UE is crucial in order to produce joint-symbols that belong to a jointconstellation from which it is possible to unambiguously recover the transmitted data of all UEs.

At the BS, after removing the $\mathrm{CP}$ and performing the DFT, the received signal at the $k$-th subcarrier, $n$-th OFDM symbol and $v$-th antenna can be expressed as

$$
\begin{gathered}
y_{k, n}^{v}=\sum_{u=1}^{U} \sqrt{\beta_{u}} h_{k, n}^{u, v} x_{k, n}^{u}+w_{k, n}^{v}, \\
1 \leq v \leq V, \quad 1 \leq k \leq K, \quad 1 \leq n \leq N,
\end{gathered}
$$




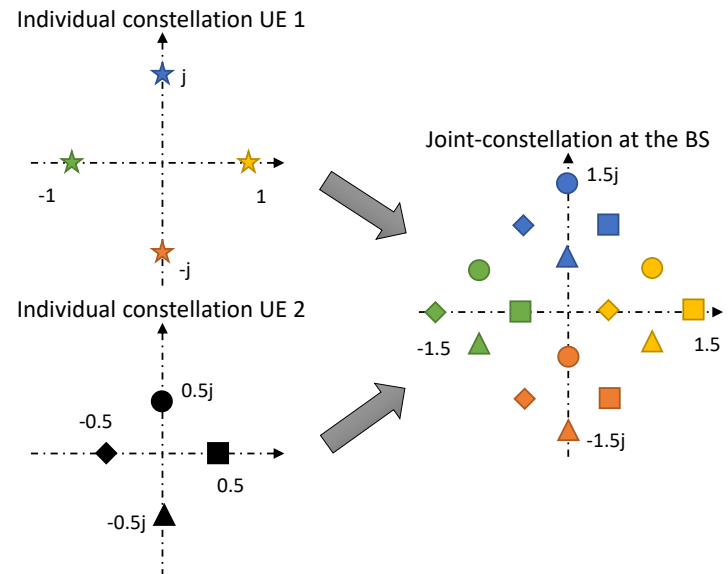

Type B

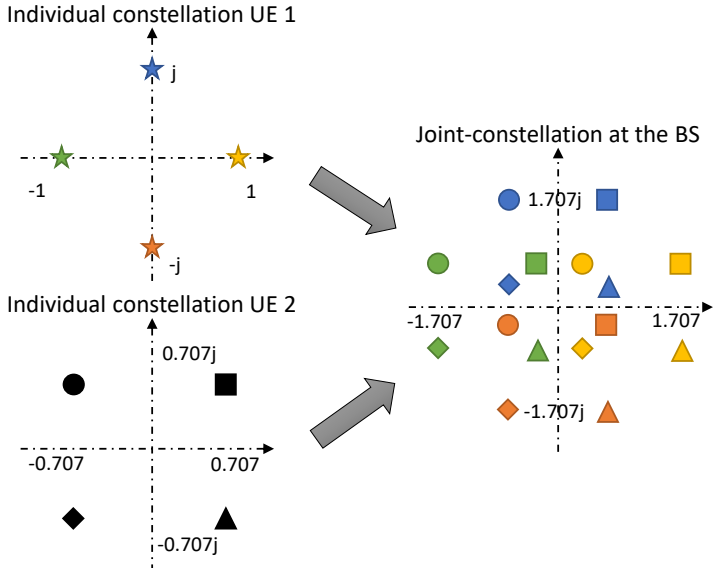

Equal Error Protection (EEP)

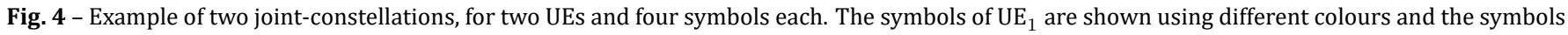
of $\mathrm{UE}_{2}$ are plotted using different markers.

where $\beta_{u}$ is the average power of the signal of the $u$-th UE, $w_{k, n}^{v}$ denotes the additive white Gaussian noise (AWGN) at $k$-th subcarrier, $n$-th OFDM symbol and $v$-th antenna, distributed as $\mathcal{C} \mathcal{N}\left(0, \sigma_{w}^{2}\right)$; and $h_{k, n}^{u, v}$ corresponds to the channel frequency response between the $u$-th UE and the $v$-th antenna at the $k$-th subcarrier and $n$-th OFDM symbol, distributed as $\mathcal{C} \mathcal{N}(0,1)$. For simplicity, we assume here that the channel response is spatially uncorrelated, while we will use more realistic channel models for performance evaluation. Besides, note that the difference in $\beta_{u}$ among different UEs may be due to the constellation design or to different propagation path loss. In the latter case, an accurate power control must be implemented to compensate this difference.

According to [12], $y_{k, n}^{v}$ is fed to the differential decoder and averaged over the spatial dimension as

$$
\begin{gathered}
z_{k, n}=\frac{1}{V} \sum_{v=1}^{V}\left(y_{k-1, n}^{v}\right)^{*} y_{k, n}^{v}, \\
2 \leq k \leq K, \quad 1 \leq n \leq N
\end{gathered}
$$

where $(\bullet)^{*}$ is the complex conjugate operation and $z_{k, n}$ denotes the received joint-symbol at the $k$-th subcarrier and $n$-th OFDM symbol. When the number of antennas is large enough and making use of the Law of Large Numbers, $z_{k, n}$ can be approximated as

$$
\begin{gathered}
z_{k, n} \stackrel{V \rightarrow \infty}{\longrightarrow} s_{k, n}=\sum_{u=1}^{U} \beta_{u} s_{k, n}^{u} \\
2 \leq k \leq K, \quad 1 \leq n \leq N,
\end{gathered}
$$

where $s_{k, n}$ is the joint-symbol at the $k$-th subcarrier and $n$-th OFDM symbol. Note that the interference and noise terms are averaged out thanks to the large number of antennas at the BS, otherwise the performance may be degraded. More details are given in [12] - [17].

The performance of the overall multi-user systems depends on the constellation of the joint-symbol. This joint- symbol must be properly designed to enable the demodulation of the transmitted information by all the UEs. Consequently, the choice of the individual constellation is crucial to produce a robust joint-constellation against interference and noise effects. The most used constellations are the Type B [12] and equal error protection (EEP) [13]. The constellation of the $u$-th UE can be expressed as

$$
\begin{gathered}
\mathcal{M}_{B}^{u}=\left\{\sqrt{\beta_{u}} \exp \left(i \frac{2 \pi}{M} m\right) \mid 0 \leq m \leq M-1\right\}, \\
\mathcal{M}_{E}^{u}=\left\{\exp \left(i\left(\frac{2 \pi}{M} m+\frac{\pi}{2 U} u\right)\right) \mid 0 \leq m \leq M-1\right\},
\end{gathered}
$$

for Type B and EEP, respectively, where $M$ denotes the number of symbols in the constellation. Fig.4 shows an illustrative example of these two types of jointconstellations, designed for the particular case of UL with only two UEs, each of them using a 4-DPSK. In the first case, all UEs have the same 4-DPSK constellation and are distinguished with a different amplitude. This produces the joint-constellation also shown in the same figure, where we can see that all symbols are equally spaced providing a robustness against possible interference and noise terms. However, those UEs with a lower amplitude will obtain a worse performance for the same noise conditions as compared to the stronger ones. Indeed, the average distance of the symbols of UE $u=1$ (shown in different colours) is much larger than the distance of UE $u=2$ (plotted in different markers). In EEP, both UEs have the same amplitude, and then the same performance. Their constellations differ in a rotation of $45^{\circ}$. However, this option presents several symbols of the joint-constellation (those placed in the middle) that are too close to each other, degrading the performance.

The design of optimal individual constellations for multiuser NCDS that work well in realistic channel conditions is still a very challenging topic, due to the diverse effects of the channel impairments and interference and the difficulty to analyse them. 


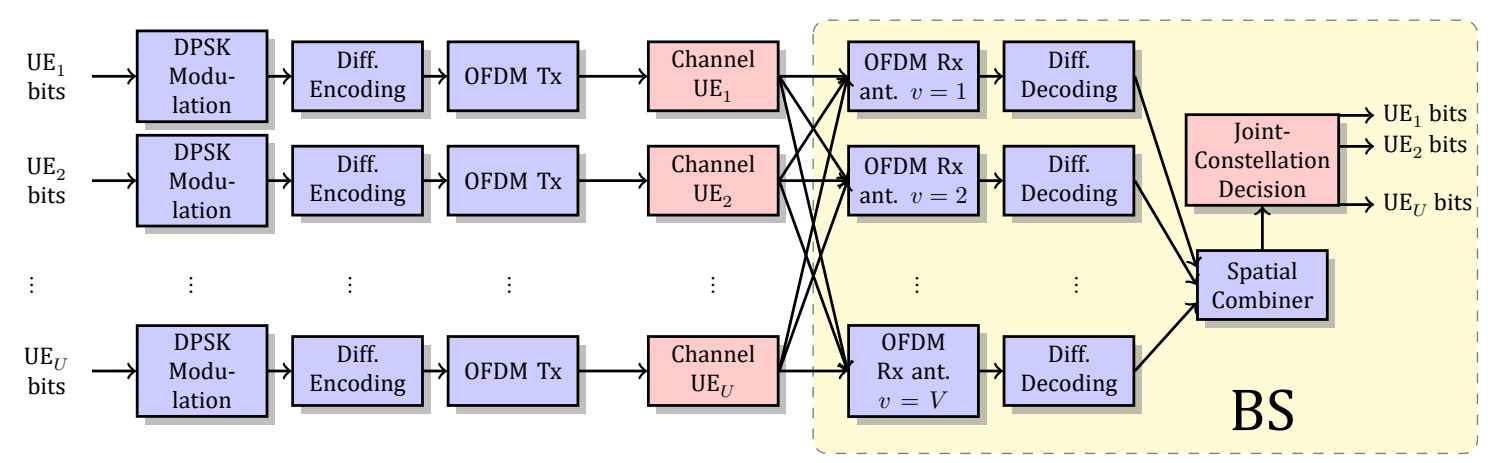

Fig. 5 - Block diagram for UL, where different UEs are multiplexed in the constellation domain.

The block diagram of a UL system addressing a more general multi-user case is shown in Fig. 5, from [17]. In this system, several UEs map their data bits into complex symbols that belong to a specific DPSK constellation. Then, the differentially encoded symbols are transmitted into an OFDM signal through a multi-path channel. The received signal at each antenna of the BS after the OFDM receiver corresponds to a superposition of different signals coming from each UE. Later, these differential symbols are non-coherently combined by using a differential decoder and averaged over the spatial domain in order to obtain the desired joint-symbol. Exploiting the spatial diversity in order to reduce the noise and multi-user interference (MUI) is crucial to obtaining a right decision from the joint-symbol [12]. Additionally, the constellation design can be combined with channel coding, where the soft information can either improve the performance or reduce the number of required antennas at the BS [16] [32].

\section{NCDS WITH MASSIVE MIMO FOR THE DOWNLINK}

Now turning to the DL, the massive number of antennas at the BS are used for transmission and we can only count on a few antennas at the UE receivers, just one per UE in many cases. The mapping schemes and multi-user constellations proposed for the UL are still valid, while we need a few more ingredients to make these schemes suitable for the DL. We explain in the following these key ingredients for a combination of NCDS with beamforming based on [19], where the BS is simultaneously transmitting the $U$ data streams through its $V$ antennas, while the UEs are receiving with their single-antenna device.

\subsection{Beamforming}

The exploitation of the diversity from the transmitter without the knowledge of the CSI is still a challenge. Due to the fact that techniques based on block codes [27] failed to exploit the large number of antennas at the transmitter, we propose the use of beamforming in order to take advantage of the massive number of antennas of the BS at the expense of using some (reduced) channel knowledge. Then, it is assumed that the angular positions of each UE are obtained through a beam management procedure to point the beams towards each UE. Once this is achieved, the data information is sent over a non-coherently processed link. Reference [18] proposed a similar idea assuming, however, an ideal case where the MUI is completely mitigated by the beamforming. Meanwhile, the combination of NCDS with a practical beamforming technique is proposed in [19], taking into account the residual MUI.

In [19] the beam-management procedure defined in 5G [1] is suggested to be performed as a first step. This procedure is responsible for accurately determining the angle of the spatial clusters of the propagation channel contributing to the signal of each UE, by transmitting some reference signals. These reference signals are specifically the synchronization signals (SS) and channel state information-reference signals (CSI-RS). The former are used when a UE would like to enter the system for the first time, while the latter are exploited for updating the angular position of an existing UE in the system.

Then, the BS transmits one or several differential data streams to each UE by using beamforming [34] as

$$
\begin{gathered}
x_{k, n}^{u, v}=b_{k, n}^{u, v} x_{k, n}^{u}, \quad 1 \leq v \leq V, \\
1 \leq u \leq U, \quad 1 \leq k \leq K, \quad 1 \leq n \leq N,
\end{gathered}
$$

where $b_{k, n}^{u, v}$ is the precoding coefficient for the $u$-th UE and $v$-th antenna of the BS, placed at the $k$-th subcarrier and $n$-th OFDM symbol. This precoding coefficient is obtained according to the estimated angular positions of each UE, and thus, it is in charge of focusing the energy in the obtained specific directions. In this way, the path loss is compensated and the MUI that results from spatially multiplexing the UEs in different beams is avoided. Similarly, beamforming can be used in the UL for the BS to receive the signal coming from these spatial directions.

Depending on the angular position of different UEs, it may be difficult to completely remove the MUI by exploiting the beamforming. Therefore, the overall performance critically depends on the scheduler which is capable of properly selecting those UEs to be simultaneously served in the same time and frequency resources and minimizing the negative impact of the mentioned MUI. Even though we are making use of some reference signals to perform 
the beamforming, any other additional overhead used in the CDS, such as the demodulation-reference signals (DM-RS), is avoided, increasing the spectral efficiency.

\subsection{Frequency diversity}

Due to the usually limited number of antennas at the UE, averaging in any dimension other than space (e.g. in time or frequency) is proposed in [19] to provide an additional source of diversity. This diversity is needed in order to obtain the required SINR gain for a good performance of the NCDS [12]. It is particularly needed if we want to multiplex several UEs in the constellation domain or enable services that are critical in terms of performance. The use of the frequency dimension is described in [17], with the advantage that each OFDM symbol can be independently processed. The proposed scheme can be easily extended to averaging either in time (processing several consecutive OFDM symbols) or space (increasing the number of receive antennas of the $\mathrm{UE}$, when possible).

The way to leverage frequency diversity consists in transmitting the same differential complex symbol in several frequency resources. At the transmitter, after performing the differential encoding, the $Q$ differential symbols are repeated as

$$
\begin{gathered}
x_{k, n}^{u}=x_{q, n}^{u} \mid q=\bmod (k-1, Q)+1, \quad K=Q \times F \\
1 \leq u \leq U, \quad 1 \leq k \leq K, \quad 1 \leq n \leq N
\end{gathered}
$$

where $F$ is the frequency repetition/averaging factor. At the receiver, analogously to $((6))$, the frequency diversity is exploited in the non-coherent detection, where the received data in the subcarriers that carry the same transmitted data are averaged as

$$
\begin{gathered}
z_{q, n}=\frac{1}{F} \sum_{f=0}^{F-1}\left(y_{q-1+f Q, n}^{v}\right)^{*} y_{q+f Q, n}^{v} \\
2 \leq q \leq Q, \quad 1 \leq n \leq N
\end{gathered}
$$

With this scheme there is a trade-off between overhead and robustness. We will see that for some particular scenarios with high mobility, even with the added overhead, this scheme will outperform the CDS in terms of throughput.

In Fig. 6 the block diagram of the system proposed in [19] is shown, combining the beamforming with the NCDS. At the receiver, assuming single-antenna devices, only frequency diversity is exploited in order to reduce the noise and MUI terms.

\section{PERFORMANCE DISCUSSION}

In this section we illustrate the performance of the combination of NCDS with a large number of antennas by discussing some numerical results. A comparison with some CDS counterparts is also provided.

\subsection{Simulation parameters}

To show some illustrative results, the numerology of the OFDM signal is chosen according to $5 \mathrm{G}$ [1]. The carrier spacing is $\Delta f=30 \mathrm{KHz}$, which is the most frequent value in different bands [35]. The bandwidth of the system is $B W=100 \mathrm{MHz}$ and the carrier frequency is $f_{c}=3.5$ GHz. The BS is equipped with a uniform linear array (ULA) of $V=128$ antenna elements, which are simultaneously serving two UEs $(U=2)$ in the whole bandwidth. Their angular separation corresponds to $72^{\circ}$ and the path loss is not considered, since the power control is assumed to work perfectly. We adopt a geometric channel model, which corresponds to a spatially correlated channel, where the power delay profile corresponds to the Type B given in [35], the delay spread is $D S=16$ ns and the angular spread is $A S=5^{\circ}$. We assume that there is a Doppler shift of $f_{d}=1.6 \mathrm{KHz}$ which corresponds approximately to a speed of $v=500 \mathrm{~km} / \mathrm{h}$ at the mentioned carrier frequency. We assume a perfect timefrequency synchronization and power control at the receiver. For the sake of space we do not provide any results for a higher carrier frequency. However, the chosen delay and angular spread can be also representative of the propagation at higher frequencies, and the same Doppler frequency would correspond to a smaller speed. Hence, the conclusions obtained with these numerical results, in particular those including beamforming (which would be mandatory to compensate the path loss), can be extrapolated to other higher frequency bands, such as $\mathrm{mm}$-Wave [36]. The SNR is conventionally defined as the ratio of the received signal power over the noise power at each antenna of the receiver.

For a baseline CDS system to compare the performance, we adopt the pilot configuration specified for the demodulation reference signals (DM-RS) in 5G [1]. In the time domain, due to the high mobility, we set four reference symbols for each slot, which corresponds to the maximum pilot density allowed by the standard. In the frequency domain, we assume the configuration Type-1, where each half of the subcarriers are allocated to each UE: the even subcarriers are used for the channel estimation of the $\mathrm{UE}_{1}$ and the odd subcarriers are for $\mathrm{UE}_{2}$. At the receiver, the channel estimation is firstly obtained at the pilot symbol resources by applying least squares (LS) [37]. Then, these estimates are interpolated to the entire resource grid by using spline interpolation.

Moreover, some hardware impairments are also considered, such as the effects of PN and the non-linear HPA. The effect of the PN is due to the instability of the local oscillators, that can only be reduced by making a more expensive one. The PN is typically modelled according to a classical Wienner random walk process given in [23], where the system performance is related to the phase noise increment variance $\sigma_{\eta}^{2}$ over the sample period $T$, where $\sigma_{\eta}^{2}=2 \pi B_{\eta} T$, with $B_{\eta}$ equal to the 3 -dB bandwidth of the Lorentzian power density carrier spectrum. The negative effect of the PN not only will degrade the received 


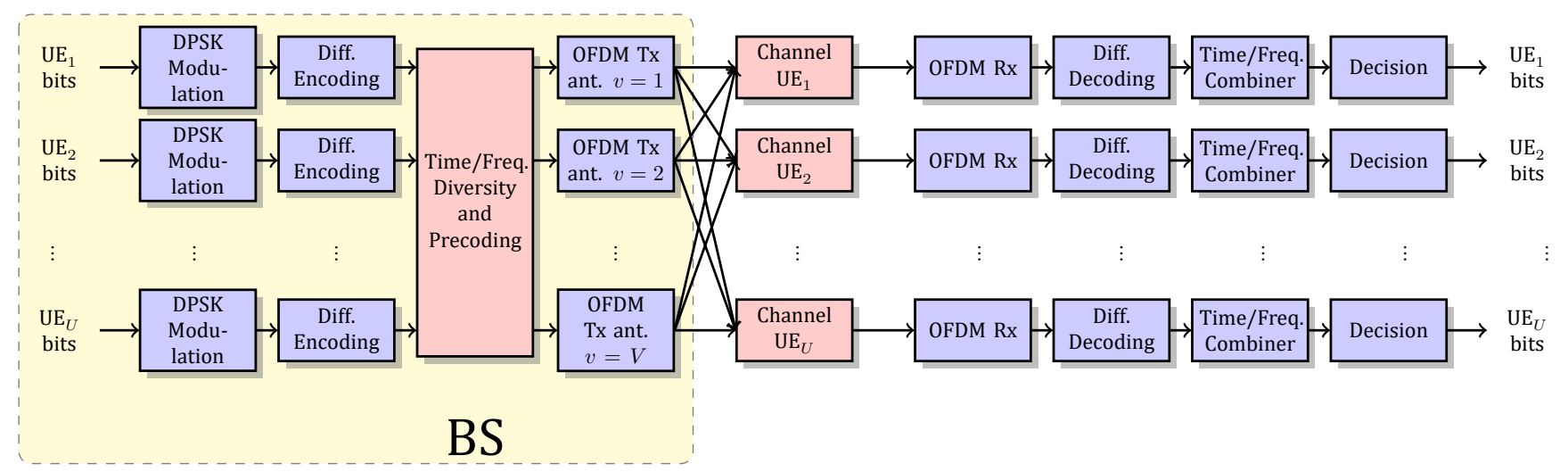

Fig. 6 - Block diagram for DL, where the BS uses a beamforming technique and all UEs are single-antenna devices.

symbols, but it will also add a common phase error [23]. The variance of the PN corresponds to $\sigma_{\eta}^{2}=10^{-5} \mathrm{rad}^{2}$. On other hand, the realistic transfer function of the HPA is not a linear function for all possible input values. This implies that the output might be saturated for those input values that are higher than the saturation point. This non-linear effect will not only degrade the quality of the received signal, but it will also enhance the out-of-band emissions. According to [24], we consider a solid state power amplifier whose output back-off (OBO) is $\mathrm{OBO}=8$ dB.

\subsection{Numerical results}

In Fig. 7, we show the SER comparison between CDS and NCDS for the UL. The constellations of the two UEs are QPSK for CDS and EEP for NCDS, both using two bits per symbol. The CDS performs a post-equalizer at the BS based on a ZF criterion. In the absence of PN and HPA, the NCDS outperforms the traditional CDS by almost two orders of magnitude of SER for moderate and high SNR scenarios. When hardware impairments are considered, the PN and HPA effects do not significantly degrade the performance of NCDS. On the other hand, the performance of CDS with and without the effect of the HPA is very poor, and it is even worse with the PN. The PN does not affect our proposed system due to the use of a differential modulation and the fact that the phase noise does not change between two contiguous subcarriers [23]. The negative effect of the HPA is negligible in both systems because the OBO is enough, in view of the robustness of the PSK signals, which are amplified separately at the transmitter of each UE.

In Fig. 8, we plot the SER comparison between CDS and NCDS for the DL. The same beamforming is considered for CDS and NCDS to spatially multiplex the two UEs. Additionally, a frequency averaging of factor $F=16$ is performed in both schemes to leverage diversity and improve the overall performance, which would be otherwise compromised. Again, in the absence of PN and HPA, the NCDS outperforms the CDS by several orders of magnitude, showing that the frequency averaging is able to ef- fectively reduce its SER, while it is not enough for CDS to work properly. When hardware impairments are considered, the performance of NCDS is degraded by both HPA and PN effects. In the same way as for the UL, we can see that NCDS is very robust to the PN effects due to the differential modulation. However, the non-linear HPA significantly degrades its performance. In this case the BS is simultaneously transmitting the signals of the two UEs and, consequently, the constant envelope characteristic of each of the PSK signals is lost when they are combined. It turns out that now the OBO is not enough and some of the signal peaks are clipped. This affects equally to both NCS and CDS. In Fig. 9, a comparison in terms of throughput is provided for the DL, whose expression is given by

$$
T_{r}=\log _{2}(M)(1-S E R) \frac{B W}{F} .
$$

We can see that even with the overhead due to a very high frequency averaging factor $(F=16)$, the NCDS still outperforms the traditional CDS. This difference is even higher when either PN or HPA effects are considered. Therefore, the throughput reduction due to the overhead produced by the frequency diversity is negligible as compared to the small throughput achieved by the CDS due to a poor performance obtained even with a large overhead.

\section{CONCLUSION}

We have provided a detailed description of the novel combination of NCDS and multi-user MIMO-OFDM based on a differential modulation scheme. Both DL and UL scenarios are considered and the performance is analyzed for realistic channel conditions including the effect of the PN and HPA.

It is shown that for channels with high mobility, the NCDS outperforms the traditional CDS obtaining a better performance, even more noticeable when PN and non-linear HPA effects are taken into account. NCDS does not require any additional PN estimation and equalization since it is inherently robust to these effects. Moreover, it shows a similar degradation with the non-linear effects of the HPA to that suffered by CDS, since they share the sensitivity of OFDM to these effects. 


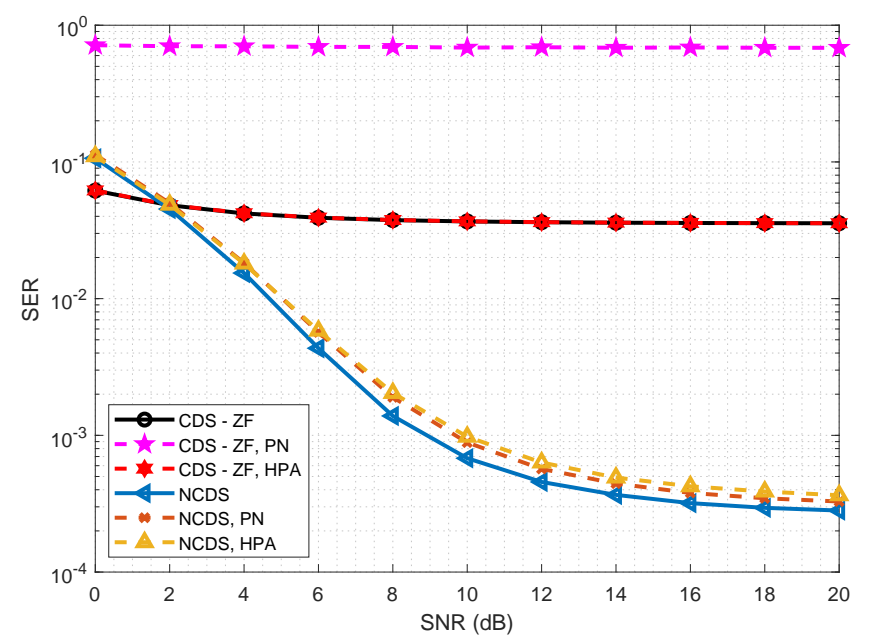

Fig. 7 - SER comparison for UL.

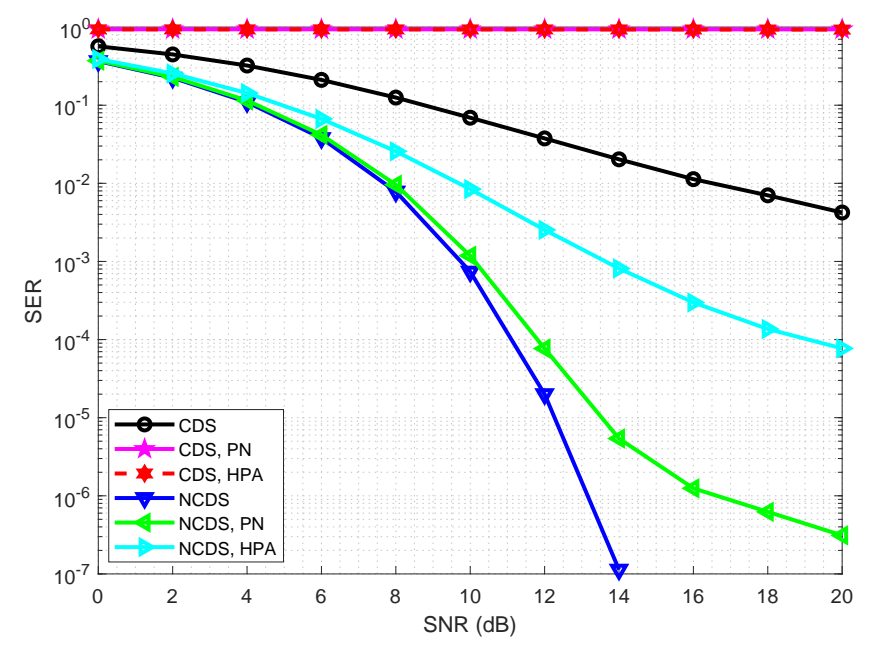

Fig. 8 - SER comparison for DL.

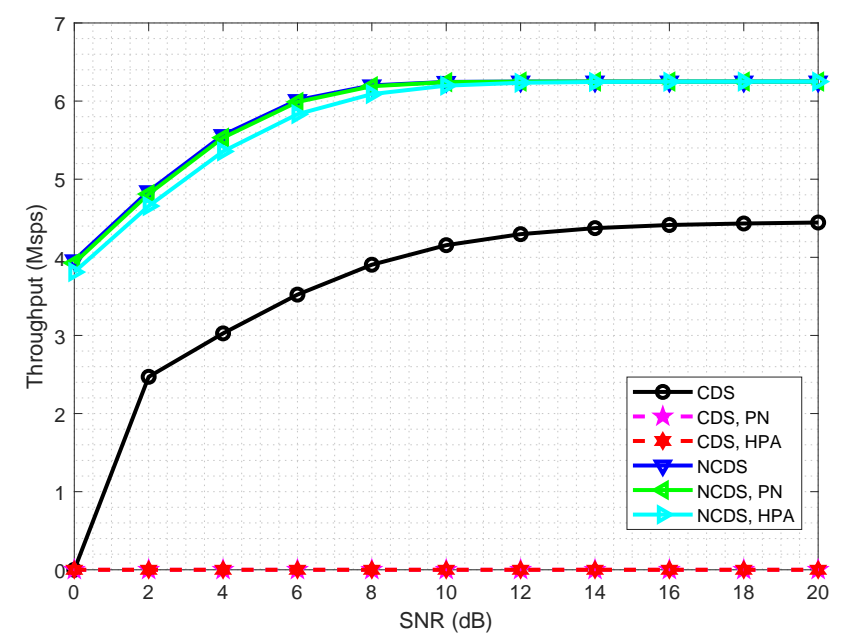

Fig. 9 - Throughput comparison for DL.

The NCDS combined with multi-user MIMO-OFDM is shown to be a feasible and very attractive technique which substantially improves the performance of the coherent systems, especially in challenging scenarios such as systems with realistic propagation channels and high mobility. However, there is still need for new research and ideas to improve the NCDS. In particular, the multiplexing of UEs in the constellation domain is not efficient, so an excessively high number of antennas is currently needed to multiplex more than two UEs [12]. There is a need to find new constellation designs that overcome this limitation. A possible way to obtain these optimal designs is to use some artificial intelligence techniques in order to automatically deal with the channel and multi-user interference effects. Not only the phases, but also the constant amplitudes of the constellations are possible values to select, giving a more complex search space, where artificial intelligence can help.

Additionally, we have seen that the performance is very sensitive to the spatial separation of the UEs that are multiplexed, either in constellation or space. Therefore, scheduling algorithms that take this into account and optimize a particular performance metric are also crucial.

The advantages of NCDS with respect to CDS vanish when the channel is quasi-static and with high SNR. Then, it is advisable to find hybrid schemes, such as [20] where the best of both paradigms is used according to the communication scenario and needs.

Finally, it is foreseen that in the future communications will be tightly integrated with sensing, which is one of the main objectives of the the Sixth Generation (6G) of mobile communications [38]. In these new systems, the efficient exploitation of CSI under a variety of scenarios will play an important role, and hence, we forecast that the exploitation of non-coherent techniques will be an interesting alternative, in order to increase the efficiency of the overall system. We hope that this review of the NCDS characteristics, feasible implementation and performance will stimulate new research and advances in this topic.

\section{ACKNOWLEDGEMENT}

This work has been funded by project TERESA-ADA (TEC2017-90093-C3-2-R) (MINECO/AEI/FEDER, UE). The authors would like to thank Ignasi Piqué-Muntané for his help in the elaboration of some figures.

\section{REFERENCES}

[1] NR; Physical channels and modulation (Release 16). Technical report, 3GPP, France, 2020.

[2] W. Guo, W. Zhang, P. Mu, F. Gao, and H. Lin. High-mobility wideband massive MIMO communications: Doppler compensation, analysis and scaling laws. IEEE Transactions on Wireless Communications, 18(6):3177-3191, June 2019.

[3] Y. Ge, W. Zhang, F. Gao, S. Zhang, and X. Ma. Beamforming network optimization for reducing channel time variation in high-mobility massive MIMO. IEEE Transactions on Communications, 67(10):6781-6795, Oct. 2019.

[4] M. Gao, B. Ai, Y. Niu, W. WU, P. Yang, F. Lyu, and X. Shen. Efficient hybrid beamforming with anti- 
blockage design for high-speed railway communications. IEEE Transactions on Vehicular Technology, 2020.

[5] G. L. Stuber, J. R. Barry, S. W. McLaughlin, Ye Li, M. A. Ingram, and T. G. Pratt. Broadband MIMO-OFDM wireless communications. Proceedings of the IEEE, 92(2):271-294, Feb. 2004.

[6] B. Yang, Z. Yu, J. Lan, R. Zhang, J. Zhou, and W. Hong. Digital beamforming-based massive MIMO transceiver for $5 \mathrm{G}$ millimeter-wave communications. IEEE Transactions on Microwave Theory and Techniques, 66(7):3403-3418, July 2018.

[7] R. A. Smith. The relative advantages of coherent and incoherent detectors: a study of their output noise spectra under various conditions. Proceedings of the IEE - Part III: Radio and Communication Engineering, 98(55):401-406, Sep. 1951.

[8] D. Middleton. Statistical theory of signal detection. Transactions of the IRE Professional Group on Information Theory, 3(3):26-51, March 1954.

[9] M. L. Doelz, E. T. Heald, and D. L. Martin. Binary data transmission techniques for linear systems. Proceedings of the IRE, 45(5):656-661, May 1957.

[10] A. Manolakos, M. Chowdhury, and A. J. Goldsmith. CSI is not needed for optimal scaling in multiuser massive SIMO systems. In 2014 IEEE International Symposium on Information Theory, pages 31173121, June 2014.

[11] M. Chowdhury, A. Manolakos, and A. Goldsmith. Scaling laws for noncoherent energy-based communications in the simo mac. IEEE Transactions on Information Theory, 62(4):1980-1992, April 2016.

[12] A. G. Armada and L. Hanzo. A non-coherent multi-user large scale SIMO system relaying on Mary DPSK. In 2015 IEEE International Conference on Communications (ICC), pages 2517-2522, June 2015.

[13] V. M. Baeza, A. G. Armada, M. El-Hajjar, and L. Hanzo. Performance of a non-coherent massive SIMO MDPSK system. In 2017 IEEE 86th Vehicular Technology Conference (VTC-Fall), pages 1-5, Sep. 2017.

[14] V. M. Baeza, A. G. Armada, W. Zhang, M. El-Hajjar, and L. Hanzo. A non-coherent multiuser largescale SIMO system relying on M-ary DPSK and BICMID. IEEE Transactions on Vehicular Technology, 67(2):1809-1814, Feb. 2018.

[15] V. M. Baeza and A. G. Armada. Non-coherent massive SIMO system based on M-DPSK for Rician channels. IEEE Transactions on Vehicular Technology, 68(3):2413-2426, March 2019.
[16] V. M. Baeza and A. G. Armada. Noncoherent massive MIMO. In Wiley $5 G$ Ref: The Essential $5 G$ Reference Online, chapter 10, pages 266-290. John Wiley \& Sons, Ltd., 2019.

[17] K. Chen-Hu and A. G. Armada. Non-coherent multiuser massive MIMO-OFDM with differential modulation. In ICC 2019 - 2019 IEEE International Conference on Communications (ICC), pages 1-6, May 2019.

[18] S. Bucher, G. Yammine, R. F. H. Fischer, and C. Waldschmidt. A noncoherent massive MIMO system employing beamspace techniques. IEEE Transactions on Vehicular Technology, 68(11):1105211063, Nov. 2019.

[19] K. Chen-Hu, Y. Liu, and A. G. Armada. Non-coherent massive MIMO-OFDM down-link based on differential modulation. IEEE Transactions on Vehicular Technology, 2020. (In press).

[20] M. J. Lopez-Morales, K. Chen-Hu, and A. GarciaArmada. Differential data-aided channel estimation for up-link massive SIMO-OFDM. IEEE Open Journal of the Communications Society, 1:976-989, 2020.

[21] F. Adachi. Adaptive differential detection for Mary DPSK. IEE Proceedings - Communications, 143(1):21-28, Feb. 1996.

[22] R. Corvaja and A. G. Armada. Phase noise degradation in massive MIMO downlink with zero-forcing and maximum ratio transmission precoding. IEEE Transactions on Vehicular Technology, 65(10):80528059, Oct. 2016.

[23] H. Ghozlan and G. Kramer. Models and information rates for wiener phase noise channels. IEEE Transactions on Information Theory, 63(4):2376-2393, April 2017.

[24] E. Costa, M. Midrio, and S. Pupolin. Impact of amplifier nonlinearities on ofdm transmission system performance. IEEE Communications Letters, 3(2):37-39, Feb. 1999.

[25] L. Lampe, R. Schober, and M. Jain. Noncoherent sequence detection receiver for Bluetooth systems. IEEE Journal on Selected Areas in Communications, 23(9):1718-1727, Sep. 2005.

[26] C. Wang, C. Huang, J. Huang, C. Chang, and C. Li. Zigbee 868/915-mhz modulator/demodulator for wireless personal area network. IEEE Transactions on Very Large Scale Integration (VLSI) Systems, 16(7):936-939, July 2008.

[27] J. Cabrejas, S. Roger, D. Calabuig, Y. M. M. Fouad, R. H. Gohary, J. F. Monserrat, and H. Yanikomeroglu. Non-coherent open-loop MIMO communications over temporally-correlated channels. IEEE Access, 4:6161-6170, 2016. 
[28] B. M. Hochwald and W. Sweldens. Differential unitary space-time modulation. IEEE Transactions on Communications, 48(12):2041-2052, Dec. 2000.

[29] V. Tarokh and H. Jafarkhani. A differential detection scheme for transmit diversity. IEEE Journal on Selected Areas in Communications, 18(7):1169-1174, July 2000.

[30] M. Beko, J. Xavier, and V. A. N. Barroso. Noncoherent communication in multiple-antenna systems: Receiver design and codebook construction. IEEE Transactions on Signal Processing, 55(12):57035715, Dec. 2007.

[31] R. H. Gohary and T. N. Davidson. Noncoherent MIMO communication: Grassmannian constellations and efficient detection. IEEE Transactions on Information Theory, 55(3):1176-1205, March 2009.

[32] F. Adachi. Reduced-state Viterbi differential detection using a recursively estimated phase reference for M-ary DPSK. IEE Proceedings - Communications, 142(4):263-270, Aug. 1995.

[33] N. Prasad, S. Wang, and X. Wang. Efficient receiver algorithms for DFT-spread OFDM systems. IEEE Transactions on Wireless Communications, 8(6):3216-3225, June 2009.

[34] J. Lota, S. Sun, T. S. Rappaport, and A. Demosthenous. 5G uniform linear arrays with beamforming and spatial multiplexing at $28,37,64$, and $71 \mathrm{ghz}$ for outdoor urban communication: A two-level approach. IEEE Transactions on Vehicular Technology, 66(11):9972-9985, Nov. 2017.

[35] Study on channel model for frequencies from 0.5 to $100 \mathrm{GHz}$ (Release 16). Technical report, 3GPP, France, 2019.

[36] T. S. Rappaport, R. W. Heath, R. C. Daniels, and J. N. Murdock. Millimeter wave wireless communications. Prentice Hall, 2015.

[37] J. Lin. Least-squares channel estimation for mobile ofdm communication on time-varying frequencyselective fading channels. IEEE Transactions on Vehicular Technology, 57(6):3538-3550, Nov. 2008.

[38] K. B. Letaief, W. Chen, Y. Shi, J. Zhang, and Y. A. Zhang. The roadmap to 6G: AI empowered wireless networks. IEEE Communications Magazine, 57(8):8490, Aug. 2019.

\section{AUTHORS}

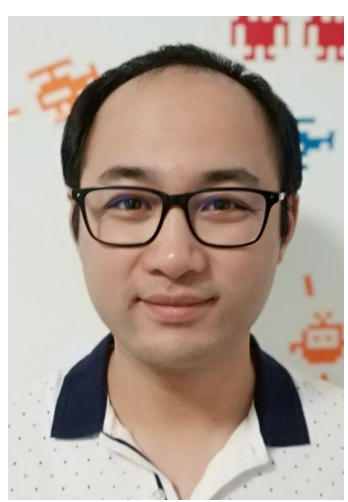

Kun Chen-Hu received his Ph.D. degree in Multimedia and Communications in 2019 from Universidad Carlos III de Madrid (Spain). Currently, he is a post-doctoral researcher in the same institution. He was awarded by UC3M in 2019 in recognition of his outstanding professional career after graduation. He visited Eurecom (France) and Vodafone Chair TU Dresden (Germany), both as guest researcher. He also participated in different research projects in collaboration with several top companies in the area of mobile communications. His research interests are related to signal processing techniques, such as waveforms design, non-coherent massive MIMO and channel estimation.

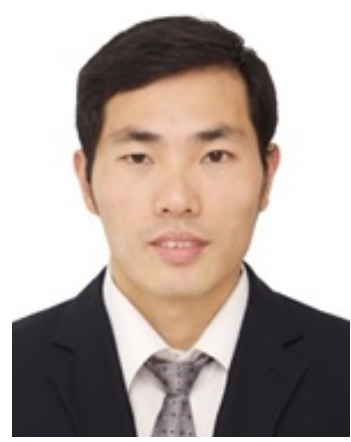

Yong Liu received a Ph.D in electronic engineering from the Department of Electric Engineering, Shanghai Jiao Tong University, Shanghai, China, in 2012. He is now with the wireless network RAN research department in Huawei Technologies Co.,Ltd., Shanghai, China. His current research interests lie in the area of $5 \mathrm{G}$ and $5 \mathrm{G}+\mathrm{MIMO}$ communication and $\mathrm{AI}$ assisted wireless networks.. 


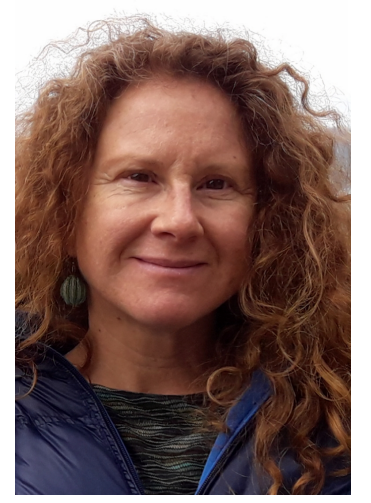

Ana García Armada (S'96-A'98-M'00-SM'08) received a $\mathrm{Ph} . \mathrm{D}$. degree in electrical engineering from the Polytechnical University of Madrid in February 1998. She is currently a Professor at University Carlos III of Madrid, Spain. She is leading the Communications Research Group at this university. She has been a visiting scholar at Stanford University, Bell Labs and University of Southampton. She has participated (and coordinated most of them) in more than 30 national and 10 international research projects, as well as 20 contracts with the industry, all of them related to wireless communications. She is the co-author of eight book chapters on wireless communications and signal processing. She has published around 150 papers in international journals and conference proceedings and she holds four patents. She has contributed to international standards organizations, such as ITU and ETSI, and is a member of the expert group of the European 5G PPP and member of the advisory committee 5JAC of the ESA as an expert appointed by Spain on 5G. She has served on the editorial boards of Physical Communication (2008-2017), IET Communications (2014-2017). She has been serving on the editorial board of IEEE Communications Letters since 2016 (Editor until Feb 2019, Senior Editor from Mar 2019, Exemplary Editor Award 2017 and 2018) and IEEE Transactions on Communications since 2019. She has served on the TPC of more than 40 conferences and she has been a member of the organizing committee of IEEE Globecom 2019, IEEE Vehicular Technology Conference (VTC) Fall 2018, Spring 2018 and 2019 and IEEE 5G Summit 2017, among others. She will be the General Chair of Globecom 2021. She was the Newsletter Editor of the IEEE ComSoc Signal Processing and Consumer Electronics Committee (2017-2018) and is now the Secretary of this committee (since 2019). She has been the Secretary of the IEEE ComSoc Women in Communications Engineering Standing Committee (2016-2017) and the Chair of this committee (2018-2019). She has received the Young Researchers Excellence Award, the Award to Outstanding achievement in research, teaching and management and the Award to Best Practices in Teaching, all from University Carlos III of Madrid, She was awarded the third place Bell Labs Prize 2014 for shaping the future of information and communications technology. She received the Outstanding service award from the IEEE ComSoc Signal Processing and Communications Electronics (SPCE) tech- nical committee in 2019. Her main interests are multicarrier and multi-antenna techniques and signal processing applied to wireless communications. 